\title{
Salvianolic acid B reverses multidrug resistance in HCT-8/VCR human colorectal cancer cells by increasing ROS levels
}

\author{
PIAOTING GUO $^{1 *}$, SONGPO WANG $^{1}$, WEI LIANG ${ }^{1 *}$, WENJING WANG $^{2}$, \\ HUIJUN WANG ${ }^{1}$, MIAOMIAO ZHAO ${ }^{1}$ and XIAOWEI LIU ${ }^{1}$ \\ ${ }^{1}$ Department of Traditional Chinese Medicine, Shanghai General Hospital, Shanghai Jiao Tong University School of Medicine; \\ ${ }^{2}$ Department of Gynecology, Shuguang Hospital Affiliated with Shanghai University of T.C.M, Shanghai 200240, P.R. China
}

Received December 11, 2015; Accepted October 17, 2016

DOI: $10.3892 / \mathrm{mmr} .2016 .6049$

\begin{abstract}
Salvianolic acid B (SalB) a water-soluble phenolic compound, extracted from Salvia miltiorrhiza, has previously been demonstrated to reverse tumor multidrug resistance (MDR), including in colorectal cancer. Reactive oxygen species (ROS) are oxygen radicals generated during aerobic metabolism (superoxide and hydroxyl radicals) and superoxide easily generating free radicals $\left(\mathrm{H}_{2} \mathrm{O}_{2}\right)$. The concept that increased ROS levels can lead to augmented tumor cell-sensitivity to chemotherapy drugs has become notable. The aim of the present study was to elucidate the role of ROS in mediating the effect of SalB on drug resistance and the correlation with drug resistance-associated protein, $\mathrm{P}$-glycoprotein (P-gp), and apoptosis-associated proteins, B-cell lymphoma 2 (Bcl-2) and Bcl-2-associated X (Bax). In the current study, through utilizing the multidrug resistant colorectal cancer cell line, HCT-8/VCR, it was demonstrate that SalB reversed MDR in HCT-8/VCR. In addition, SalB significantly increased ROS levels, which may have accelerated the apoptosis of HCT-8/VCR cells by downregulating Bcl-2 and increasing Bax protein expression. Furthermore the increased intracellular ROS levels may have inhibited P-gp expression at the gene and protein levels. In conclusion, the data of the current study demonstrate that SalB reversed MDR in HCT-8/VCR cells, and the effect is associated with increased ROS levels, which may downregulate P-gp expression and promote tumor cell apoptosis, which in turn increases the sensitivity of drug-resistant cells to chemotherapy drugs.
\end{abstract}

Correspondence to: Dr Songpo Wang, Department of Traditional Chinese Medicine, Shanghai General Hospital, Shanghai Jiao Tong University School of Medicine, 100 Haining Road, Shanghai 200240, P.R. China

E-mail: 13386259791@163.com

${ }^{*}$ Contributed equally

Key words: colorectal cancer, multidrug resistance, salvianolic acid $\mathrm{B}$, reactive oxygen species, $\mathrm{P}$-glycoprotein

\section{Introduction}

Colorectal cancer is a gastrointestinal malignancy, and the third most common cause of cancer and cancer-associated mortality (1). The 5-year survival rate of colorectal cancer patients is $50-55 \%$ (2). Chemotherapy is a primary therapeutic strategy for colorectal cancer; however, multidrug resistance (MDR) is a key reason for the failure of this treatment. Identification of effective MDR reversal strategies is therefore required to address this issue.

Reactive oxygen species (ROS), which are primarily produced by mitochondria, have strong reactivity and a short life cycle, and may damage the majority of organelles, including mitochondria. Previously, ROS were considered to be toxic substances that cause cell damage and play an important role in the occurrence and development of tumors. However, in recent years, anti-tumor effects exerted by ROS have been demonstrated. Multiple studies have demonstrated that long-term administration of a drug may reduce intracellular ROS levels and convert sensitive tumor cells into drug-resistant cells (3). ROS concentrations moderately greater than physiological levels downregulate P-glycoprotein (P-gp) expression and increase tumor cell sensitivity to chemotherapy drugs (4). In addition, ROS induce apoptosis of tumor cells via various pathways, increasing their chemotherapeutic sensitivity $(5,6)$.

The B-cell lymphoma 2 (Bcl-2) family is critical in the mitochondrial apoptotic pathway; Bcl-2 (anti-apoptotic) and Bcl-2-associated X (Bax; pro-apoptotic) are important members of this family. The mitochondria-dependent apoptosis pathway is a primary pathway of apoptosis, and mitochondrial membrane potential decline is a feature of early apoptosis (7). Bax translocates from the cytoplasm to mitochondria and associates with the mitochondrial membrane, to promote the opening of the mitochondrial permeability transition pore, which leads to the loss of mitochondrial membrane potential and the destruction of membrane integrity, thereby promoting the release of mitochondrial pro-apoptotic factors and inducing cell apoptosis. The pro-apoptotic effect of Bax may be inhibited by overexpression of Bcl-2. P-gp, an important member of the ABC transporter superfamily, is encoded by the MDR1 gene and functions as an energy-dependent drug efflux pump. P-gp-mediated drug efflux is the classical 
mechanism underlying MDR. In colorectal cancer, P-gp expression levels and frequencies are high, with up to $96 \%$ of cells expressing this molecule (8). A previous study has revealed that $\mathrm{P}$-gp function in MDR colorectal cancer cells is significantly enhanced, and that P-gp serves an important role in the generation and maintenance of colorectal cancer drug resistance (9).

Salvianolic acid B (SalB) is a water-soluble phenolic compound, extracted from Salvia miltiorrhiza. Its role in reversing tumor MDR has attracted increasing attention $(10,11)$. In the present study, SalB was used to treat the MDR colorectal cancer cell line HCT-8/VCR, and explore its effect on drug resistance. The present study aimed to determine the potential mechanism by which SalB reverses MDR in colorectal cancer by assessing its effects on ROS levels, P-gp expression and the levels of apoptosis-associated factors in HCT-8/VCR.

\section{Materials and methods}

Drugs and reagents.HCT-8 and HCT-8/VCR, human colorectal cancer sensitive and multidrug resistant cell lines, respectively, were purchased from Gu Haibo Biological Technology Co., Ltd. (Shanghai, China). SalB was obtained from Shanghai Winherb Medical Technology Co., Ltd. (Shanghai, China). Vincristine (VCR) was manufactured by Shenzhen Main Luck Pharmaceuticals Inc. (Shenzhen, China). 5-Fluorouracil (5-FU), cisplatin (CDDP) and $N$-acetylcysteine (NAC) were purchased from Sigma-Aldrich (Merck Millipore, Darmstadt, Germany). Paclitaxel (Taxol) was from Bristol-Myers Squibb (New York, NY, USA). $\mathrm{H}_{2} \mathrm{O}_{2}$ was obtained from Sinopharm Chemical Reagent Co., Ltd. (Shanghai, China). Cell Counting Kit-8 (CCK-8) was manufactured by Dojindo Molecular Technologies, Inc. (Kumamoto, Japan). Bicinchoninic acid (BCA) assay and ROS kits were from Beyotime Institute of Biotechnology (Haimen, China). A rabbit monoclonal antibody against P-gp (catalog no. ab170904) was purchased from Abcam (Cambridge, MA, USA). Rabbit monoclonal antibodies against Bcl-2 (catalog no. 4223), Bax (catalog no. 5023) and GAPDH (catalog no. 2118), and the horseradish peroxidase-conjugated secondary antibody mouse anti-rabbit IgG (catalog no. 5127), were obtained from Cell Signaling Technology, Inc. (Danvers, MA, USA).

Cell culture. HCT-8 and HCT-8/VCR cells were cultured in RPMI-1640 medium (Gibco; Thermo Fisher Scientific, Inc., Waltham, MA, USA) containing 10\% fetal bovine serum (Gibco; Thermo Fisher Scientific, Inc.), $100 \mu \mathrm{g} / \mathrm{ml}$ penicillin and $100 \mu \mathrm{g} / \mathrm{ml}$ streptomycin. VCR $(800 \mathrm{ng} / \mathrm{ml})$ was added in the culture medium for HCT-8/VCR cells to maintain drug resistance. Cells were incubated at $37^{\circ} \mathrm{C}$ in a humid environment containing $5 \% \mathrm{CO}_{2}$. The VCR in the medium was removed 1 week before experiments were performed, and cells in the logarithmic phase were used.

Assessment of drug resistance in HCT-8/VCR cells by CCK-8. HCT-8 and HCT-8/VCR cells in the logarithmic phase were seeded at $5 \times 10^{3} / \mathrm{ml} /$ well $(100 \mu \mathrm{l})$ in 96 -well plates. Chemotherapy drugs, VCR, 5-FU, CDDP and Taxol, at five different concentrations $(0.01,0.1,1,10$ and $100 \mu \mathrm{g} / \mathrm{ml})$, were added following overnight incubation. Three replicates were performed for each condition, and $10 \mu \mathrm{lCCK}-8$ was added after $48 \mathrm{~h}$ incubation. After $40 \mathrm{~min}$, absorbance was read by using a microplate reader at $450 \mathrm{~nm}$, with a reference wavelength of $650 \mathrm{~nm}$. The following equation was used to calculate growth inhibition: Cell suppression rate $(\%)=(1-\mathrm{OD}$ in the study group/OD in the control group) $\mathrm{x} 100$. The experiment was repeated 3 times. SPSS 18.0 (SPSS, Inc., Chicago, IL, USA) was employed to calculate the median inhibitory concentration $\left(\mathrm{IC}_{50}\right)$ of each drug. The cell resistance index was calculated as follows: resistance index $(\%)=\mathrm{IC}_{50}$ resistant cells $/ \mathrm{IC}_{50}$ parent cells $x 100$.

Assessment of the cytotoxic effect of SalB on HCT-8/VCR cells by $C C K-8$. Inhibition rates were calculated as described above. Then, drug concentration and cell inhibition rate were used as ordinates to plot a dose-response curve of SalB for HCT-8/VCR. $\mathrm{IC}_{50}$ and non-toxic dose $\left(\mathrm{IC}_{20}\right)$ were derived from the curve. The experiment was repeated three times.

Evaluation of reverse index for drug resistance of SalB in HCT-8/VCR by CCK-8. $\mathrm{IC}_{50}$ values for VCR, 5-FU, CDDP and Taxol for HCT-8/VCR cells were calculated as described above. In addition, the $\mathrm{IC}_{50}$ values of their combination with SalB for HCT-8/VCR cells were also calculated. Reverse index for drug resistance was calculated as follows, with the experiment repeated 3 times. Reverse index $=\mathrm{IC}_{50}$ before combination $/ \mathrm{IC}_{50}$ after combination.

Detection of ROS levels by flow cytometry (FCM). HCT-8 and HCT-8/VCR cells in the logarithmic phase were divided into 8 groups: Including HCT-8, HCT- 8 + NAC, HCT-8/VCR, HCT-8/VCR $+\mathrm{H}_{2} \mathrm{O}_{2}, \mathrm{HCT}-8 / \mathrm{VCR}+\mathrm{SalB} 20$, HCT-8/VCR + SalB 40, HCT-8/VCR + SalB 80 and HCT-8/VCR + SalB80 + NAC. The HCT-8/VCR + $\mathrm{H}_{2} \mathrm{O}_{2}$ group served as a positive control. Cells were pretreated with the ROS scavenger NAC $(5 \mathrm{mM})$, and the exogenous activator $\mathrm{H}_{2} \mathrm{O}_{2}(200 \mu \mathrm{M})$ for $2 \mathrm{~h}$ prior to SalB treatment. Cells were subsequently cultured for $48 \mathrm{~h}$ at $37^{\circ} \mathrm{C}$ with $5 \% \mathrm{CO}_{2}$. Dichlorofluorescin diacetate (DCFH-DA; $1 \mathrm{ml}$ ) diluted with serum free RPMI-1640 culture medium was added to cells, which were cultured at $37^{\circ} \mathrm{C}$ for $30 \mathrm{~min}$ and washed with PBS three times to remove free DCFH-DA. Then, cells were harvested and resuspended in PBS for FCM analysis using a FACSCalibur flow cytometer (BD Biosciences, Franklin Lakes, NJ, USA). Untreated cells were used as controls; 10,000 cells were detected in each sample, using average fluorescence intensity to reflect intracellular ROS levels. Data were analyzed using FlowJo software version 10.0.7 (FlowJo, LLC, Ashland, OR). The experiment was repeated three times.

Effect of SalB on HCT-8/VCR apoptosis rate. FCM was performed with cells divided into 6 groups: HCT-8/VCR, HCT-8/VCR + SalB20, HCT-8/VCR + SalB40, HCT-8/VCR + SalB80, HCT-8/VCR + SalB80 + NAC, and HCT-8/VCR $+\mathrm{H}_{2} \mathrm{O}_{2}$ groups. They were treated as described above for $48 \mathrm{~h}$ at $37^{\circ} \mathrm{C}$ with $5 \% \mathrm{CO}_{2}$. After washing, cells were resuspended in PBS to $1 \times 10^{6}$ cells $/ \mathrm{ml}$. To $100 \mu \mathrm{l}$ cell suspensions, $5 \mu 1$ Annexin V-fluorescein isothiocyanate and $5 \mu \mathrm{l}$ propidium iodide were added. The mixture was gently 
shaken and incubated at room temperature, and shielded from direct sunlight for $15 \mathrm{~min}$. FCM was performed immediately after washing. The experiment was repeated three times.

Effect of SalB on MDR1 mRNA expression in HCT-8/VCR. HCT-8/VCR cells in the logarithmic phase were divided into 6 groups: HCT-8/VCR, HCT-8/VCR $+\mathrm{H}_{2} \mathrm{O}_{2}$, HCT-8/VCR + SalB20, HCT-8/VCR + SalB40, HCT-8/VCR + SalB80 and HCT-8/VCR + SalB80 + NAC. The HCT-8/VCR $+\mathrm{H}_{2} \mathrm{O}_{2}$ group served as a positive control. For reverse transcription-quantitative polymerase chain reaction (RT-qPCR), cells were seeded at $5 \times 10^{5} / \mathrm{ml}$. Total RNA was extracted using the RNeasy Extraction kit (catalog no. 9108Q; Takara Biotechnology Co., Ltd., Dalian, China), and $1 \mu \mathrm{g}$ total RNA was reverse-transcribed to cDNA using a FastQuant RT kit (catalog no. KR106; Tiangen Biotech Co., Ltd., Beijing, China). Thermocycling conditions for RT were as follows: An initial step at $42^{\circ} \mathrm{C}$ for $15 \mathrm{~min}$ followed by $95^{\circ} \mathrm{C}$ for $3 \mathrm{~min}$. qPCR on the obtained cDNA was performed in triplicate using the $\mathrm{SYBR}^{\circledR}{ }^{\circledR}$-Green PCR Master mix (catalog no. FP302; Tiangen Biotech Co., Ltd.), with thermocycling conditions as follows: An initial denaturation step at $95^{\circ} \mathrm{C}$ for $2 \mathrm{~min}$, followed by 40 cycles of $95^{\circ} \mathrm{C}$ for $10 \mathrm{sec}$ and $60^{\circ} \mathrm{C}$ for $30 \mathrm{sec}$. The quantitation cycle $(\mathrm{Cq})$ values of each sample were calculated and normalized to GAPDH, which served as an internal control, using the $2^{-\Delta \Delta C q}$ method (12). The primers used are listed in Table I.

Influence of SalB on $\mathrm{P}$-gp, $\mathrm{Bcl}-2$ and Bax protein expression in HCT-8/VCR. The protein expression levels were detected by western blotting. Grouping and treatments were the same as apoptosis analysis. Cytoplasmic and nuclear proteins were extracted using radioimmunoprecipitation assay lysis buffer (catalog no. P0013B; Beyotime Institute of Biotechnology), according to the manufacturer's protocol and protein content was determined using a Bicinchoninic Acid Protein assay kit (catalog no. P0010; Beyotime Institute of Biotechnology). Total protein $(30 \mu \mathrm{g})$ was resolved by SDS-PAGE. Blocking was performed using 5\% bovine serum albumin (BSA) for $2 \mathrm{~h}$ at room temperature following membrane transfer. Anti-P-gp, anti-Bcl-2, anti-Bax and anti-GAPDH antibodies [all diluted 1:1,000 with 5\% BSA in TBS containing $0.1 \%$ Tween-20 (TBST)] were added and incubated overnight at $4^{\circ} \mathrm{C}$. Following three washes with TBST, membranes were probed with a horseradish peroxidase-conjugated secondary antibody $(1: 1,000)$ for $2 \mathrm{~h}$ at $37^{\circ} \mathrm{C}$. Membranes were washed three times with TBST, and protein bands were visualized using an Enhanced Chemiluminiscence reagent (catalog no. P0018; Beyotime Institute of Biotechnology). Quantity-One software version 4.6.2 (Bio-Rad Laboratories, Inc., Hercules, CA, USA) was used for densitometry. Experiments were repeated three times.

Statistical analysis. Data were analyzed using SPSS 18.0 (SPSS, Inc., Chicago, IL, USA), and presented as the mean \pm standard deviation. One-way analysis of variance was used for comparisons among groups with Student-Newman-Keuls-q test used for multiple comparisons between groups. $\mathrm{P}<0.05$ was considered to indicate a statistically significant difference.
Table I. Primer sequences.

\begin{tabular}{ll}
\hline Primer & \multicolumn{1}{c}{ Sequence (5'-3') } \\
\hline MDR1 forward & CCACTCCTCCACCTTTGAC \\
MDR1 reverse & ACCCTGTTGCTGTAGCCA \\
GAPDH forward & AAGGTGAAGGTCGGAGTCAAC \\
GAPDH reverse & GGGGTCATTGATGGCAACAATA
\end{tabular}

Table II. Resistance of HCT- 8 and HCT-8/VCR cells to chemotherapy drugs.

\begin{tabular}{lcrc}
\hline & \multicolumn{2}{c}{$\mathrm{IC}_{50}(\mu \mathrm{g} / \mathrm{ml})$} & \\
\cline { 2 - 3 } Drug & HCT- 8 & HCT-8/VCR & $\begin{array}{c}\text { Resistance } \\
\text { index }\end{array}$ \\
\hline VCR & $0.76 \pm 0.16$ & $39.09 \pm 1.47^{\mathrm{a}}$ & 51.43 \\
5-FU & $0.25 \pm 0.11$ & $2.22 \pm 1.12^{\mathrm{a}}$ & 8.88 \\
CDDP & $1.81 \pm 0.19$ & $9.06 \pm 1.14^{\mathrm{a}}$ & 5.01 \\
Taxol & $0.19 \pm 0.01$ & $5.34 \pm 0.84^{\mathrm{a}}$ & 28.11 \\
\hline
\end{tabular}

${ }^{\text {a }}<0.01$ vs. HCT-8 cells. VCR, vincristine; 5-FU, 5-fluorouracil; CDDP, cisplatin; Taxol, paclitaxel.

Table III. Growth inhibitory effects of salvianolic acid B on HCT-8/VCR cells.

\begin{tabular}{lcc}
\hline Concentration $(\mu \mathrm{g} / \mathrm{ml})$ & Cell inhibition rate & $\mathrm{n}$ \\
\hline 500 & $94.47 \pm 1.42$ & 3 \\
250 & $66.66 \pm 12.34$ & 3 \\
125 & $26.37 \pm 0.37$ & 3 \\
12.5 & $15.17 \pm 4.06$ & 3 \\
1.25 & $8.50 \pm 1.64$ & 3 \\
\hline
\end{tabular}

\section{Results}

Multidrug resistance in HCT-8/VCR cells. CCK- 8 assay data (Table II) demonstrated that HCT-8/VCR were resistant to the four chemotherapy drugs (VCR, 5-FU, CDDP and Taxol), with significant differences in the $\mathrm{IC}_{50}$ values of cytotoxicity compared with the sensitive HCT-8 cells $(\mathrm{P}<0.01)$.

Cytotoxicity of SalB in HCT-8/VCR. Cytotoxicity of SalB in HCT-8/VCR was assessed by the CCK- 8 method. As demonstrated in Table III, SalB significantly inhibited HCT-8/VCR proliferation in a concentration-dependent manner in a certain concentration range. $\mathrm{IC}_{20}$ and $\mathrm{IC}_{50}$ were $20.79 \pm 4.76 \mu \mathrm{g} / \mathrm{ml}$ and $114.79 \pm 10.94 \mu \mathrm{g} / \mathrm{ml}$, respectively. Concentrations $\leq \mathrm{IC}_{20}$ were selected as non-cytotoxic dose to perform drug resistance experiments.

Reverse index of SalB for drug resistance in HCT-8/VCR. As demonstrated in Table IV, CCK-8 data demonstrated that 
Table IV. $\mathrm{IC}_{50}$ for chemotherapy drugs used in combination with salvianolic acid B in HCT-8/VCR cells.

\begin{tabular}{lccc}
\hline & \multicolumn{2}{c}{$\mathrm{IC}_{50}(\mu \mathrm{g} / \mathrm{ml})$} & \\
\cline { 2 - 3 } Drug & $\begin{array}{c}\text { Chemotherapy } \\
\text { only }\end{array}$ & $\begin{array}{c}\text { Chemotherapy }+ \text { SalB } \\
(20 \mu \mathrm{g} / \mathrm{ml})\end{array}$ & $\begin{array}{c}\text { Reversal } \\
\text { index }\end{array}$ \\
\hline VCR & $39.09 \pm 1.47$ & $2.76 \pm 0.92^{\mathrm{a}}$ & 14.16 \\
5-FU & $2.22 \pm 1.12$ & $0.26 \pm 0.10^{\mathrm{b}}$ & 8.54 \\
CDDP & $9.06 \pm 1.41$ & $2.05 \pm 0.47^{\mathrm{a}}$ & 4.42 \\
TAXOL & $5.34 \pm 0.84$ & $1.16 \pm 0.26^{\mathrm{a}}$ & 4.60 \\
\hline
\end{tabular}

${ }^{\mathrm{a}} \mathrm{P}<0.0001,{ }^{\mathrm{b}} \mathrm{P}<0.01$ vs. single medication. VCR, vincristine; 5-FU, fluorouracil; CDDP, cisplatin; Taxol, paclitaxel.
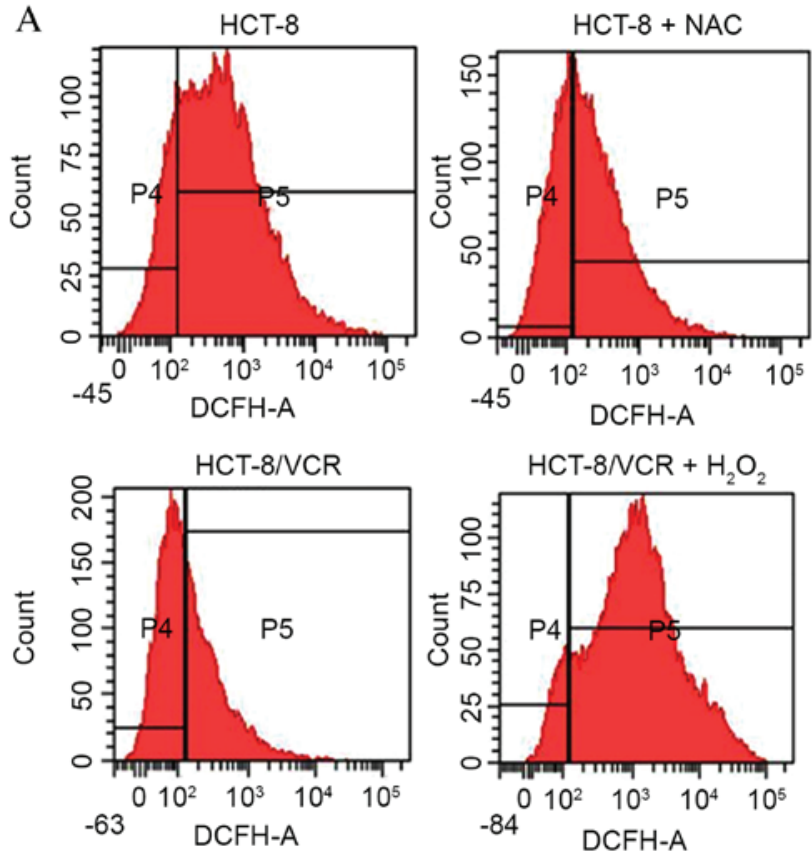

B

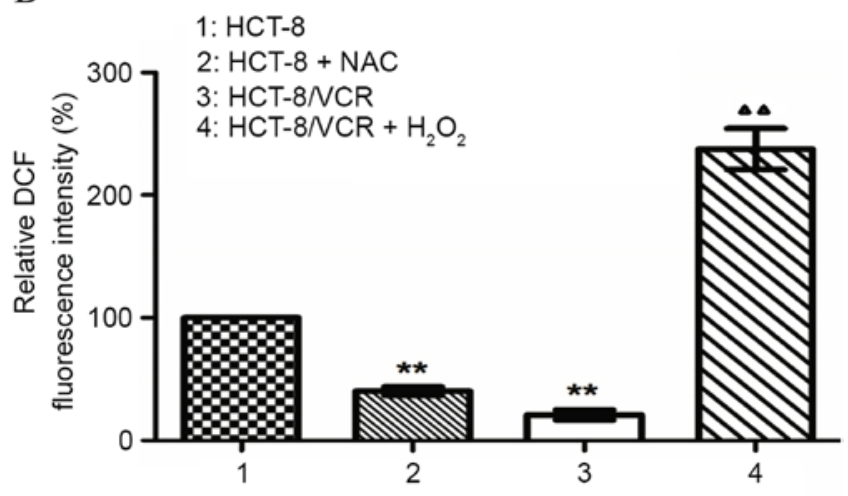

Figure 1. Changes of ROS levels in HCT-8 and HCT-8/VCR cell lines. (A) Flow cytometric histograms of DCFH fluorescence in cells and (B) quantification of flow cytometry results. Values are presented as the mean \pm standard deviation. ${ }^{* *} \mathrm{P}<0.01$ vs. HCT- 8 cells, ${ }^{\Delta \Delta} \mathrm{P}<0.01$ vs. HCT-8/VCR cells. ROS, reactive oxygen species; DCF, dichlorofluorescin; NAC, $N$-acetylcysteine.

non-cytotoxic SalB doses $\left(20 \mu \mathrm{g} / \mathrm{ml} ;<\mathrm{IC}_{20}\right)$ could significantly increase HCT-8/VCR sensitivity to VCR, 5-FU, CDDP and
Taxol, further reducing the $\mathrm{IC}_{50}$ of various chemotherapeutics. Therefore, it was inferred that SalB could reverse the MDR of colorectal cancer HCT-8/VCR cells.

Effect of SalB on intracellular ROS levels. Average fluorescence intensities of intracellular DCFH detected by FCM (Fig. 1) demonstrated that ROS levels were significantly decreased in HCT-8/VCR compared with those in HCT-8. Following HCT- 8 cell treatment with the ROS scavenger, NAC (5 mM), and HCT-8/VCR incubation with $\mathrm{H}_{2} \mathrm{O}_{2}(200 \mu \mathrm{M})$ for $2 \mathrm{~h}$, ROS level changes were analyzed by FCM. Notably, NAC significantly reduced ROS levels compared with untreated HCT-8 cells $(\mathrm{P}<0.01)$, whereas $\mathrm{H}_{2} \mathrm{O}_{2}$ markedly increased ROS compared with untreated HCT-8/VCR cells $(\mathrm{P}<0.01)$.

Following treatment with $\mathrm{SalB}$ at different concentrations (20, 40 and $80 \mu \mathrm{g} / \mathrm{ml}$ ) for $48 \mathrm{~h}$, ROS levels in HCT-8/VCR cells were significantly increased in a concentration-dependent manner. In addition, statistical significance was obtained in cells treated with $80 \mu \mathrm{g} / \mathrm{ml}$ SalB compared with the untreated HCT-8/VCR control group $(\mathrm{P}<0.01)$. Pretreatment with NAC $(5 \mathrm{mM})$ significantly decreased intracellular ROS levels in the HCT-8/VCR+SalB 80 group $(\mathrm{P}<0.01)$, suggesting that NAC eliminated the increased ROS levels caused by high concentrations of SalB (Fig. 2).

Effect of SalB on HCT-8/VCR apoptosis. As demonstrated in Fig. 3, SalB induced HCT-8/VCR apoptosis in a concentration-dependent manner. In addition, apoptosis rates in cells treated with SalB at 40 and $80 \mu \mathrm{g} / \mathrm{ml}$ (no significant difference between both groups) were significantly higher compared with the untreated control group $(\mathrm{P}<0.01)$. HCT-8/VCR apoptosis rates in cells receiving SalB after pretreatment with NAC $(5 \mathrm{mM})$ for $2 \mathrm{~h}$ were significantly lower than in the groups treated only with $80 \mu \mathrm{g} / \mathrm{ml}$ SalB $(\mathrm{P}<0.05)$.

Effect of SalB on MDR1 levels in HCT-8/VCR. As demonstrated in Fig. 4, Real-Time PCR demonstrated that following SalB low, medium and high-dose treatments for $48 \mathrm{~h}$, MDR1 expression levels were decreased in a dose-dependent manner. Furthermore, there was a statistical decrease in the MDR1 levels following SalB treatment compared with the untreated negative control group $(\mathrm{P}<0.01)$ and no difference compared with the HCT-8/VCR $+\mathrm{H}_{2} \mathrm{O}_{2}$ positive control group ( $\left.\mathrm{P}>0.05\right)$. Pretreatment with NAC partially reversed the high SalB concentration-induced decrease of MDR1 expression $(\mathrm{P}<0.01)$.

Effect of SalB on P-gp protein expression in HCT-8/VCR cells. As demonstrated in Figs. 5 and 6, SalB significantly reduced $\mathrm{P}$-gp protein expression in a dose-dependent manner $(\mathrm{P}<0.01)$, however, there was no statistical difference between the high SalB concentration $(80 \mu \mathrm{g} / \mathrm{ml})$ and positive control groups $(\mathrm{P}>0.05)$. Following pretreatment with NAC and high SalB for 48 h, P-gp expression levels were significantly increased compared with cells administered with high SalB only $(\mathrm{P}<0.01)$.

Effect of SalB on Bcl-2 and Bax protein expression levels in HCT-8/VCR. Western blot analysis (Figs. 7 and 8) demonstrated that following treatment with SalB at 20, 40 and $80 \mu \mathrm{g} / \mathrm{ml}$, Bcl-2 expression levels in HCT-8/VCR cells were decreased 

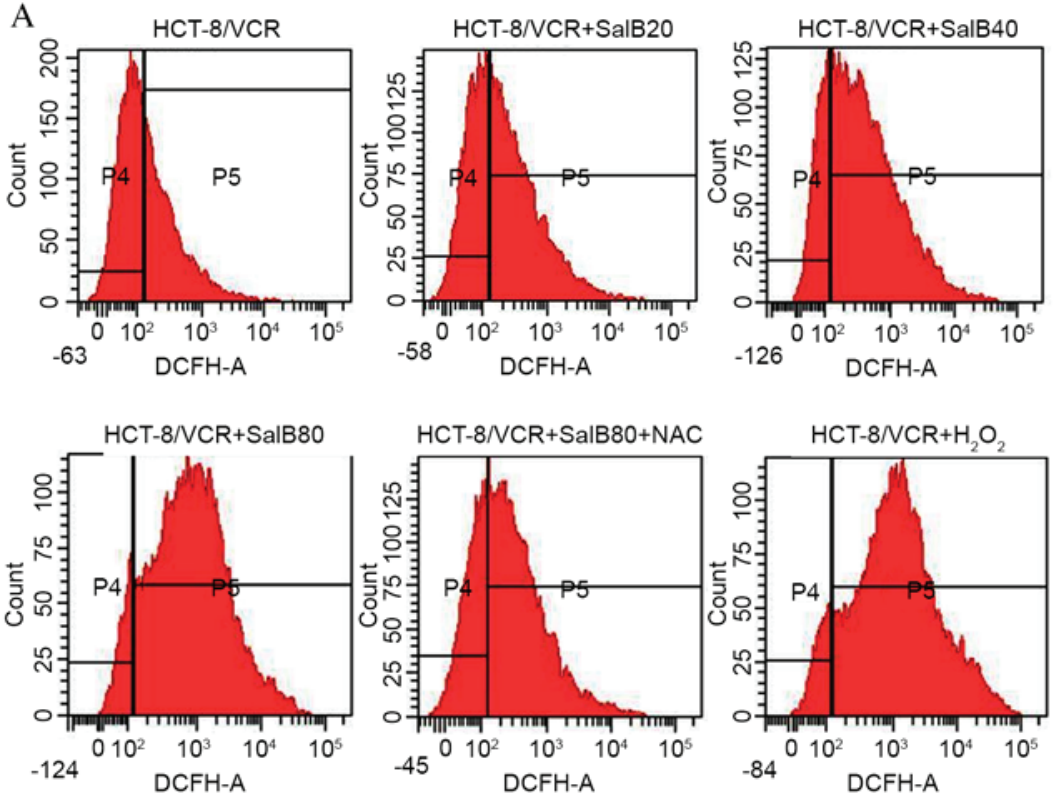

$\mathrm{B}$

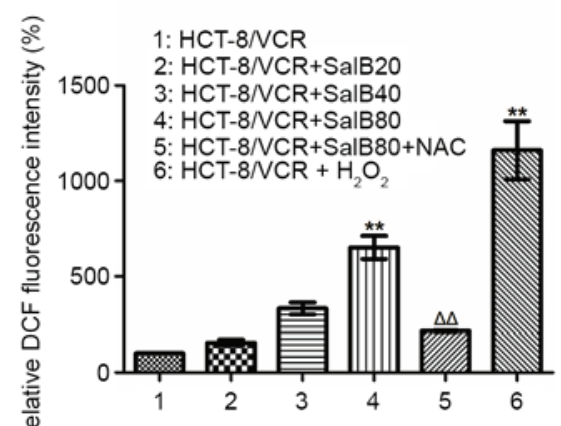

Figure 2. Effect of SalB on ROS production in HCT-8/VCR cells. (A) Flow cytometric histograms of DCF fluorescence in HCT-8/VCR cells and (B) quantification of flow cytometry results following treatment with 20,40 and $80 \mu \mathrm{g} / \mathrm{ml} \mathrm{SalB}$. Values are presented as the mean \pm standard deviation. ${ }^{* *} \mathrm{P}<0.01 \mathrm{vs}$. HCT-8/VCR cells, ${ }^{\Delta \Lambda} \mathrm{P}<0.01$ vs. HCT-8/VCR + SalB80 cells. ROS, reactive oxygen species; DCF, dichlorofluorescin; SalB, salvianolic acid B.
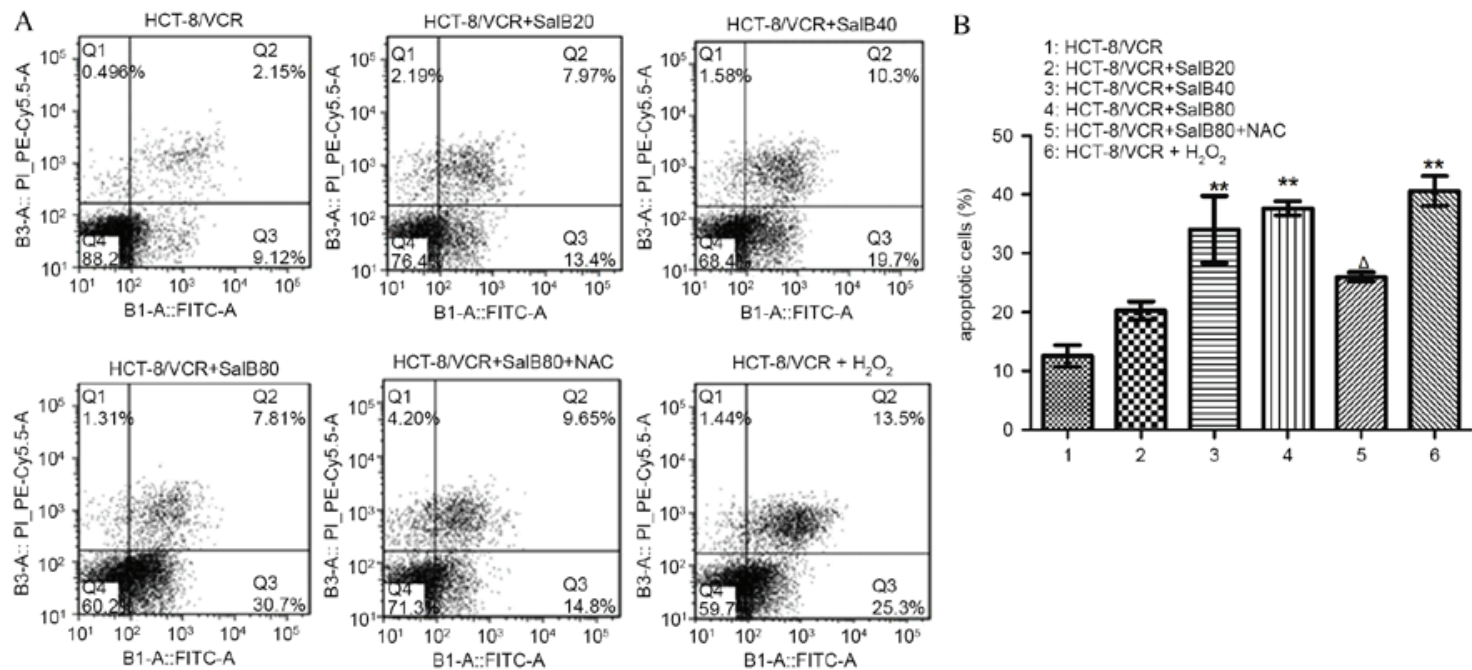

Figure 3. Effect of SalB on apoptosis in HCT-8/VCR cells. (A) Representative flow cytometric dot plots and (B) quantitation of the population of apoptotic cells following treatment with 20,40 and $80 \mu \mathrm{g} / \mathrm{ml} \mathrm{SalB}$, and NAC or $\mathrm{H}_{2} \mathrm{O}_{2}$. Values are presented as the mean \pm standard deviation. ${ }^{* *} \mathrm{P}<0.01 \mathrm{vs}$. HCT-8/VCR group, ${ }^{\Delta} \mathrm{P}<0.05$ vs. HCT-8/VCR+SalB80 group. SalB, salvianolic acid B; NAC, $N$-acetylcysteine.

compared with the negative control group. Additionally, Bax expression levels were increased by SalB compared with the negative control group. No differences in Bcl-2 and Bax expression levels were observed between the positive control and high SalB concentration group. Following pretreatment with NAC and high SalB, the Bcl-2/Bax ratio was significantly increased compared with cells administered with SalB only. Quantification of the Bcl-2/Bax ratios are presented in Fig. 8.

\section{Discussion}

Primary and secondary MDR in colorectal cancer cells is significantly higher than in other cancers, explaining the low efficacy of chemotherapy for colorectal cancer (13). Drug efflux mediated by P-gp is the classic mechanism of MDR. It has been demonstrated that P-gp downregulation or efflux inhibition can increase the sensitivity of resistant cells to chemotherapeutic drugs and reverse MDR in colorectal cancer cells (14-18). Drug resistance mediated by anti-apoptosis is also a key MDR mechanism (19). Apoptosis is also considered the intersection of MDR pathways. ROS are oxygen radicals generated during aerobic metabolism [superoxide $\left(\mathrm{O}_{2}-\right)$ and hydroxyl radicals], with superoxide easily generating free radicals (hydrogen peroxide) (20). It is known that, compared with sensitive cells, antioxidant levels in resistant cells are higher, and ROS levels are lower (21-25). Furthermore, increased ROS 


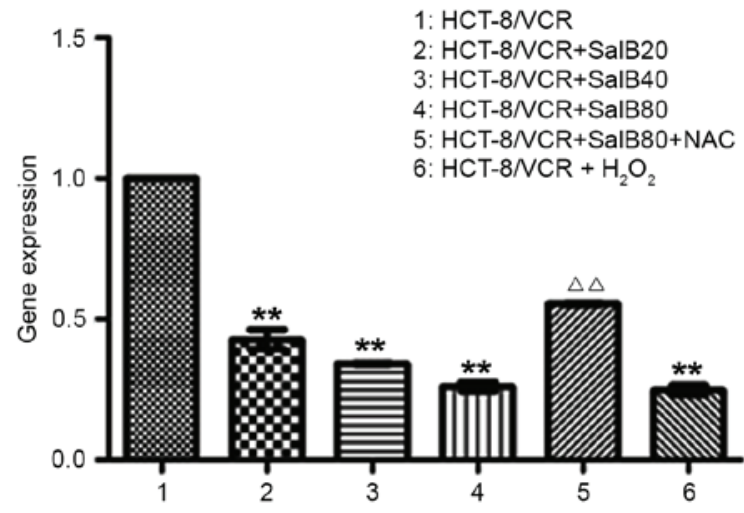

Figure 4. Reverse transcription-quantitative polymerase chain reaction for P-glycoprotein mRNA levels. HTC-8/VCR cells were treated with 20,40 and $80 \mu \mathrm{g} / \mathrm{ml} \mathrm{SalB}$, and $\mathrm{NAC}$ or $\mathrm{H}_{2} \mathrm{O}_{2}$. Values are presented as the mean \pm standard deviation. ${ }^{* *} \mathrm{P}<0.01$ vs. HCT-8/VCR group, ${ }^{\Delta \Delta} \mathrm{P}<0.01$ vs. HCT-8/VCR + SalB80 group. SalB, salvianolic acid B; NAC, $N$-acetylcysteine.

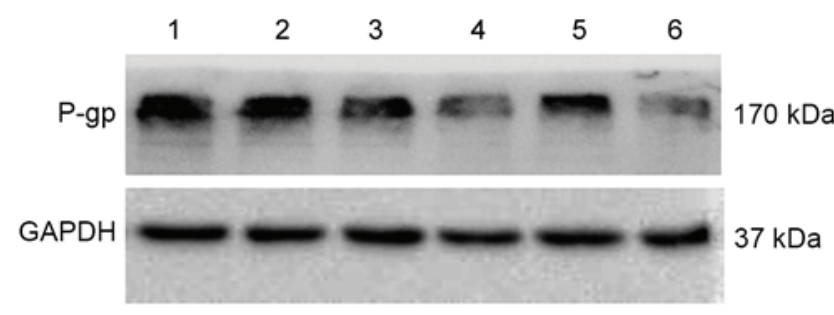

Figure 5. Protein expression of P-gp. HTC-8/VCR cells were treated with 20,40 and $80 \mu \mathrm{g} / \mathrm{ml} \mathrm{SalB}$, and $\mathrm{NAC}$ or $\mathrm{H}_{2} \mathrm{O}_{2}$. 1, HCT-8/VCR group; 2, HCT-8/VCR + SalB20 group; 3, HCT-8/VCR + SalB40 group; 4, HCT-8/VCR + SalB80 group; 5, HCT-8/VCR + SalB80 + NAC group; 6 , HCT-8/VCR $+\mathrm{H}_{2} \mathrm{O}_{2}$ group. P-gp, P-glycoprotein; SalB, salvianolic acid B; NAC, $N$-acetylcysteine.

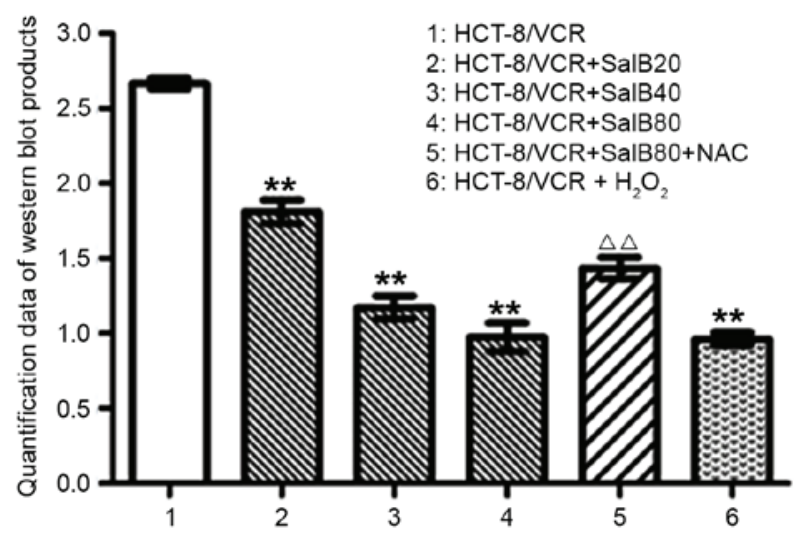

Figure 6. Quantitation of P-glycoprotein protein expression. HTC-8/VCR cells were treated with 20,40 and $80 \mu \mathrm{g} / \mathrm{ml} \mathrm{SalB}$, and $\mathrm{NAC}$ or $\mathrm{H}_{2} \mathrm{O}_{2}$. Values are presented as the mean \pm standard deviation. ${ }^{* *} \mathrm{P}<0.01$ vs. HCT- $8 / \mathrm{VCR}$ group, ${ }^{\Delta} \mathrm{P}<0.01$ vs. HCT-8/VCR + SalB80 group. SalB, salvianolic acid B; NAC, $N$-acetylcysteine.

levels lead to augmented sensitivity of tumor cells to chemotherapy drugs (26-29).

HCT-8 (sensitive) and HCT-8/VCR (resistant) colorectal cancer cells were used in the current study. It was initially demonstrated that HCT-8/VCR cells were multidrug resistant, and screened non-toxic SalB doses $\left(\leq \mathrm{IC}_{20}\right)$. Subsequently, SalB

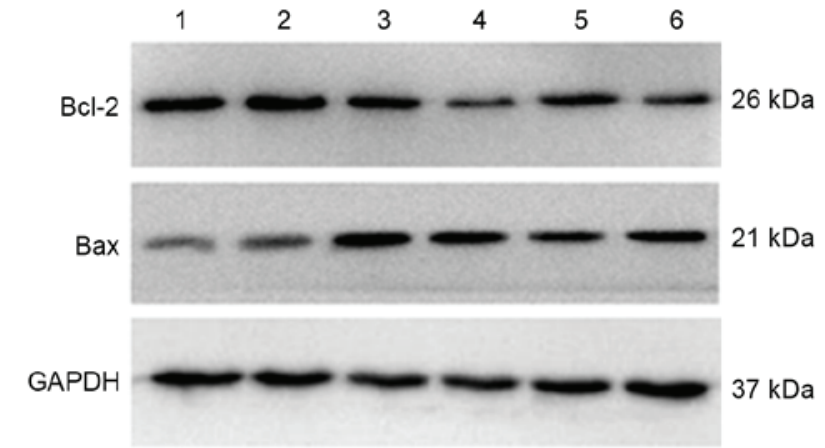

Figure 7. Effect of $\mathrm{SalB}, \mathrm{NAC}$ and $\mathrm{H}_{2} \mathrm{O}_{2}$ on the protein expression levels of Bcl-2 and Bax. HTC-8/VCR cells were treated with 20,40 and $80 \mu \mathrm{g} / \mathrm{ml}$ SalB, and NAC or $\mathrm{H}_{2} \mathrm{O}_{2} .1$, HCT-8/VCR group; 2 , HCT-8/VCR + SalB20 group; 3, HCT-8/VCR + SalB40 group; 4 , HCT-8/VCR + SalB80 group; $5, \mathrm{HCT}-8 / \mathrm{VCR}+\mathrm{SalB} 80+\mathrm{NAC}$ group; $6, \mathrm{HCT}-8 / \mathrm{VCR}+\mathrm{H}_{2} \mathrm{O}_{2}$ group. ${ }^{* *} \mathrm{P}<0.01$ vs. HCT-8/VCR group, ${ }^{\Delta \Delta} \mathrm{P}<0.01$ vs. HCT-8/VCR + SalB80 group. $\mathrm{SalB}$, salvianolic acid B; NAC, $N$-acetylcysteine. Bcl-2, Bcl-2 apoptosis regulator; Bax, Bcl-2 associated $\mathrm{X}$ apoptosis regulator.

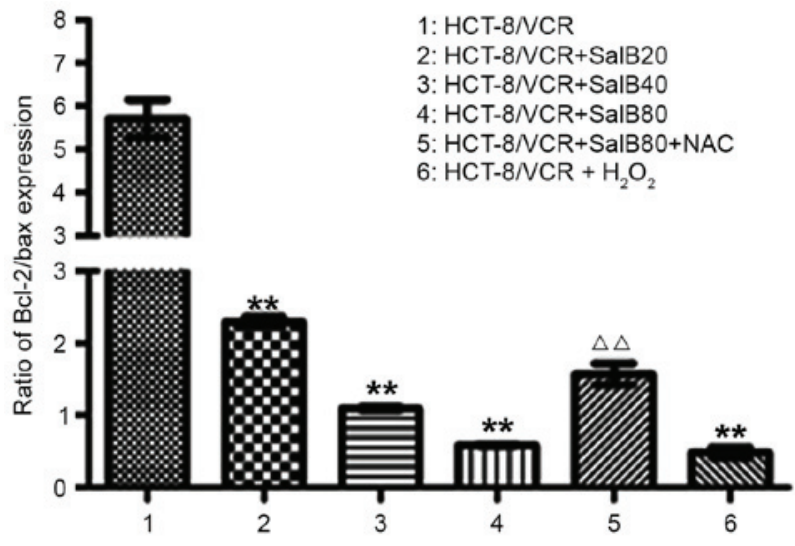

Figure 8. Effect of SalB on Bcl-2/Bax expression ratio in HCT-8/VCR cells. HTC-8/VCR cells were treated with 20,40 and $80 \mu \mathrm{g} / \mathrm{ml} \mathrm{SalB}$, and NAC or $\mathrm{H}_{2} \mathrm{O}_{2}$. Values are presented as the mean \pm standard deviation. ${ }^{* *} \mathrm{P}<0.01$ vs. HCT-8/VCR group, ${ }^{\Delta \Delta} \mathrm{P}<0.01$ vs. HCT-8/VCR + SalB 80 group. SalB, salvianolic acid $\mathrm{B}$; NAC, $N$-acetylcysteine. $\mathrm{Bcl}-2$, Bcl-2 apoptosis regulator; $\mathrm{Bax}, \mathrm{Bcl}-2$ associated $\mathrm{X}$ apoptosis regulator.

at non-toxic doses was combined with VCR, 5-FU, CDDP and Taxol, and was demonstrated that SalB reversed MDR of HCT-8/VCR.

Notably, ROS levels in HCT-8/VCR were lower than those in sensitive HCT- 8 cells as demonstrated by FCM, indicating that HCT-8/VCR drug resistance may be associated with low ROS levels, which could be increased by SalB. The current study demonstrated that NAC reversed SalB-induced apoptosis, suggesting that SalB reversed MDR in HCT-8/VCR by increasing ROS levels. Subsequently, the expression levels of apoptosis-associated proteins, and drug resistance-associated P-gp, were assessed. It was observed that changes of intracellular ROS levels may secondarily regulate the expression of $\mathrm{Bcl}-2$, Bax and $\mathrm{P}$-gp, affecting apoptosis and drug resistance.

Therefore, we speculated that SalB might reverse multidrug resistance in colorectal cancer HCT-8/VCR cells through two mechanisms related to increased ROS levels in resistant cells: i) Down-regulated P-gp at the gene and protein 
levels, which results in increased sensitivity of resistant cells to chemotherapy drugs; ii) reduced anti-apoptosis marker (Bcl-2) expression and increased pro-apoptosis (Bax) marker levels decrease mitochondrial transmembrane potential, promote apoptosis of drug resistant cells and reverse multidrug resistance of colorectal cancer HCT-8/VCR cells. More mechanisms of the colorectal cancer multidrug resistance reversed by SalB and reverse resistant effects in body require further investigation.

\section{References}

1. Siegel RL, Miller KD and Jemal A: Cancer statistics, 2016. CA Cancer J Clin 66: 7-30, 2016.

2. Chen W, Zheng R, Baade PD, Zhang S, Zeng H, Bray F, Jemal A, Yu XQ and He J: Cancer statistics in China, 2015. CA Cancer J Clin 66: 115-132, 2016.

3. Maiti AK: Genetic determinants of oxidative stress-mediated sensitization of drug-resistant cancer cells. Int J Cancer 130: 1-9, 2012.

4. Dahdouh F, Raane M, Thévenod F and Lee WK: Nickel-induced cell death and survival pathways in cultured renal proximal tubule cells: Roles of reactive oxygen species, ceramide and ABCB1. Arch Toxicol 88: 881-892, 2014.

5. Lo YL and Tu WC: Co-encapsulation of chrysophsin-1 and epirubicin in PEGylated liposomes circumvents multidrug resistance in HeLa cells. Chem Biol Interact 242: 13-23, 2015.

6. Brahimi-Horn MC, Giuliano S, Saland E, Lacas-Gervais S, Sheiko T, Pelletier J, Bourget I, Bost F, Féral C, Boulter E, et al: Knockout of Vdacl activates hypoxia-inducible factor through reactive oxygen species generation and induces tumor growth by promoting metabolic reprogramming and inflammation. Cancer Metab 3: 8, 2015.

7. Singh KK: Mitochondrial dysfunction is a common phenotype in aging and cancer. Ann N Y Acad Sci 1019: 260-264, 2004.

8. Sinicrope FA, Hart J, Brasitus TA, Michelassi F, Lee JJ and Safa AR: Relationship of P-glycoprotein and carcinombryonic antigen expression in human colon carcinoma to local invasion. DNA ploidy, and disease relapse. Caner 74: 2908-2917, 1994.

9. Salphati L, Plise EG and Li G: Expression and activity of the efflux transporters $\mathrm{ABCB} 1, \mathrm{ABCC} 2$ and $\mathrm{ABCG} 2$ in the human colorectal carcinoma cell line LS513. Eur J Pharm Sci 37: 463-468, 2009.

10. Wang M, Sun G, Wu P, Chen R, Yao F, Qin M, Luo Y, Sun H, Zhang Q, Dong X and Sun X: Salvianolic Acid B prevents arsenic trioxide-induced cardiotoxicity in vivo and enhances its anticancer activity in vitro. Evid Based Complement Alternat Med 2013: 759483, 2013

11. Zhao Y, Hao Y, Ji H, Fang Y, Guo Y, Sha W, Zhou Y, Pang X, Southerland WM, Califano JA and Gu X: Combination effects of salvianolic acid B with low-dose celecoxib on inhibition of head and neck squamous cell carcinoma growth in vitro and in vivo. Cancer Prev Res (Phila) 3: 787-796, 2010.

12. Livak KJ and Schmittgen TD: Analysis of relative gene expression data using real-time quantitative PCR and the 2(-Delta Delta C(T)) method. Methods 25: 402-408, 2001

13. Shen A, Chen H, Chen Y, Lin J, Lin W, Liu L, Sferra TJ and Peng J: Pien Tze Huang Overcomes Multidrug Resistance and Epithelial-Mesenchymal Transition in Human Colorecta Carcinoma Cells via Suppression of TGF- $\beta$ Pathway. Evid Based Complement Alternat Med 2014: 679436, 2014.
14. Ravindranath AK, Kaur S, Wernyj RP, Kumaran MN, Miletti-Gonzalez KE, Chan R, Lim E, Madura K and Rodriguez-Rodriguez L: CD44 promotes multi-drug resistance by protecting P-glycoprotein from FBXO21-mediated ubiquitination. Oncotarget 6: 26308-26321, 2015.

15. Katayama K, Noguchi K and Sugimoto Y: FBXO15 regulates $\mathrm{P}$-glycoprotein/ABCB1 expression through the ubiquitin-proteasome pathway in cancer cells. Cancer Sci 104: 694-702, 2013

16. Sui H, Liu X, Jin BH, Pan SF, Zhou LH, Yu NA, Wu J, Cai JF, Fan ZZ, Zhu HR and Li Q: Zuo Jin Wan, a Traditional Chinese Herbal Formula, Reverses P-gp-Mediated MDR In Vitro and In Vivo. Evid Based Complement Alternat Med 2013: 957078, 2013.

17. Sun H, Liu XD, Liu Q, Wang FP, Bao XQ and Zhang D: Reversal of P-glycoprotein-mediated multidrug resistance by the novel tetrandrine derivative W6. J Asian Nat Prod Res 17: 638-648, 2015.

18. Jin Y, Zhang Z, Zhao T, Liu X and Jian L: Mixed micelles of doxorubicin overcome multidrug resistance by inhibiting the expression of P-Glycoprotein. J Biomed Nanotechnol 11: 1330-1338, 2015

19. Beeran AA, Maliyakkal N, Rao CM and Udupa N: The enriched fraction of Elephantopus scaber Triggers apoptosis and inhibits multi-drug resistance transporters in human epithelial cancer cells. Pharmacogn Mag 11: 257-268, 2015.

20. Agarwal A, Ayaz A, Samanta L, Sharma R, Assidi M, Abuzenadah AM and Sabanegh E: Comparative proteomic network signatures in seminal plasma of infertile men as a function of reactive oxygen species. Clin Proteomics 12: 23, 2015.

21. Lo YL, Wang W and Ho CT: 7,3',4'-Trihydroxyisoflavone modulates multidrug resistance transporters and induces apoptosis via production of reactive oxygen species. Toxicology 302: 221-232, 2012.

22. Pandey V, Chaube B and Bhat MK: Hyperglycemia regulates MDR-1, drug accumulation and ROS levels causing increased toxicity of carboplatin and 5-fluorouracil in MCF-7 cells. J Cell Biochem 112: 2942-2952, 2011.

23. Wei N, Liu GT, Chen XG, Liu Q, Wang FP and Sun H: H1, a derivative of Tetrandrine, exerts anti-MDR activity by initiating intrinsic apoptosis pathway and inhibiting the activation of Erk1/2 and Akt1/2. Biochem Pharmacol 82: 1593-1603, 2011.

24. Singer E, Judkins J, Salomonis N, Matlaf L, Soteropoulos P, McAllister S and Soroceanu L: Reactive oxygen species-mediated therapeutic response and resistance in glioblastoma. Cell Death Dis 6: e1601, 2015.

25. Brozovic A, Ambriović-Ristov A and Osmak M: The relationship between cisplatin-induced reactive oxygen species, glutathione, and BCL-2 and resistance to cisplatin. Crit Rev Toxicol 40: 347-359, 2010.

26. Okon IS and Zou MH: Mitochondrial ROS and cancer drug resistance: Implications for therapy. Pharmacol Res 100: 170-174, 2015.

27. Okon IS, Coughlan KA, Zhang M, Wang Q and Zou MH: Gefitinib-mediated reactive oxygen specie (ROS) instigates mitochondrial dysfunction and drug resistance in lung cancer cells. J Biol Chem 290: 9101-9110, 2015.

28. Su Z, Chen M, Xiao Y, Sun M, Zong L, Asghar S, Dong M, Li H, Ping $\mathrm{Q}$ and Zhang C: ROS-triggered and regenerating anticancer nanosystem: An effective strategy to subdue tumor's multidrug resistance. J Control Release 196: 370-383, 2014.

29. Hao Y, Lin C, Fan H, Yu Y, Li N and Chen S: Proteomic analysis of Cucumis sativus cotyledons after glucohexaose treatment as a part of ROS accumulation related resistance mechanism. Proteome Sci 12: 34, 2014. 